\title{
Communication
}

\section{Bicontinuous Gyroid Phase of a Water-Swollen Wedge-Shaped Amphiphile: Studies with In-Situ Grazing-Incidence X-ray Scattering and Atomic Force Microscopy}

\author{
Kseniia N. Grafskaia 1,2®, Azaliia F. Akhkiamova ${ }^{2,3}$, Dmitry V. Vashurkin ${ }^{2,4}$, Denis S. Kotlyarskiy ${ }^{2,4}(\mathbb{D}$, \\ Diego Pontoni ${ }^{3}{ }^{(D}$, Denis V. Anokhin ${ }^{1,2}$, Xiaomin Zhu ${ }^{5,6}$ and Dimitri A. Ivanov ${ }^{1,2,4,7, *(1)}$ \\ 1 Moscow Institute of Physics and Technology, Instituskiy per. 9, 141700 Dolgoprudny, Russia; \\ grafskayaxeniya@gmail.com (K.N.G.); deniano@yahoo.com (D.V.A.) \\ 2 Institute of Problems of Chemical Physics, Russian Academy of Sciences, Chernogolovka, \\ 142432 Moscow, Russia; azigy@mail.ru (A.F.A.); dmvav21@gmail.com (D.V.V.); $9920860 @$ gmail.com (D.S.K.) \\ 3 Partnership for Soft Condensed Matter (PSCM), ESRF-The European Synchrotron, 71 Avenue des Martyrs, \\ 38043 Grenoble, France; diego.pontoni@esrf.fr \\ 4 Faculty of Chemistry, Lomonosov Moscow State University (MSU), GSP-1, 1-3 Leninskiye Gory, \\ 119991 Moscow, Russia \\ 5 DWI-Leibniz-Institute for Interactive Materials e.V., D-52056 Aachen, Germany; zhu@dwi.rwth-aachen.de \\ check for \\ updates \\ Citation: Grafskaia, K.N.; \\ Akhkiamova, A.F.; Vashurkin, D.V.; \\ Kotlyarskiy, D.S.; Pontoni, D.; \\ 6 Institute for Technical and Macromolecular Chemistry of RWTH Aachen University, Forkenbeckstr. 50, \\ D-52056 Aachen, Germany \\ 7 Institut de Sciences des Matériaux de Mulhouse-IS2M, CNRS UMR 7361, Jean Starcky 15, \\ F-68057 Mulhouse, France \\ * Correspondence: dimitri.ivanov@uha.fr
} Anokhin, D.V.; Zhu, X.; Ivanov, D.A. Bicontinuous Gyroid Phase of a Water-Swollen Wedge-Shaped Amphiphile: Studies with In-Situ Grazing-Incidence X-ray Scattering and Atomic Force Microscopy. Materials 2021, 14, 2892. https:// doi.org/10.3390/ma14112892

Academic Editors: Thomas

Walter Cornelius and

Souren Grigorian

Received: 10 May 2021

Accepted: 24 May 2021

Published: 28 May 2021

Publisher's Note: MDPI stays neutral with regard to jurisdictional claims in published maps and institutional affiliations.

Copyright: (c) 2021 by the authors. Licensee MDPI, Basel, Switzerland. This article is an open access article distributed under the terms and conditions of the Creative Commons Attribution (CC BY) license (https:/ / creativecommons.org/licenses/by/ $4.0 /)$.

\begin{abstract}
We report on formation of a bicontinuous double gyroid phase by a wedge-shaped amphiphilic mesogen, pyridinium $4^{\prime}$-[3", $4^{\prime \prime}, 5^{\prime \prime}$-tris-(octyloxy)benzoyloxy]azobenzene-4-sulfonate. It is found that this compound can self-organize in zeolite-like structures adaptive to environmental conditions (e.g., temperature, humidity, solvent vapors). Depending on the type of the phase, the structure contains 1D, 2D, or 3D networks of nanometer-sized ion channels. Of particular interest are bicontinuous phases, such as the double gyroid phase, as they hold promise for applications in separation and energy. Specially designed environmental cells compatible with grazing-incidence X-ray scattering and atomic force microscopy enable simultaneous measurements of structural parameters/morphology during vapor-annealing treatment at different temperatures. Such in-situ approach allows finding the environmental conditions at which the double gyroid phase can be formed and provide insights on the supramolecular structure of thin films at different spatial levels.
\end{abstract}

Keywords: wedge-shaped amphiphile; double gyroid phase; grazing-incidence X-ray scattering; environmental atomic force microscopy; vapor annealing

\section{Introduction}

Molecular self-assembly is a spontaneous process during which the constitutive elements self-organize under action of non-covalent bonding forces [1-3]. Among selfassembling species, amphiphilic low-molecular-weight mesogens have been extensively studied because of their fascinating phase behavior [4,5]. In particular, wedge-shaped amphiphiles can self-assemble into a remarkable range of lyotropic liquid crystalline (LLC) and thermotropic liquid crystalline (TLC) mesophases with intricate one-, two-, and threedimensional $(1 \mathrm{D}, 2 \mathrm{D}$, and 3D) periodic nanostructures [6,7]. Due to the unique structures of non-lamellar LC mesophases, they have attracted significant interest over the past few decades particularly for their applications in such areas as drug delivery [8,9], membrane protein crystallization $[10,11]$, energy conversion and storage, gas storage, chemical sensing, and others [12,13]. 
Nanoporous membranes are already widely used in various applications, like chemical separation and purification, fuel conversion, and ecology [14]. They are perspective not only for classical chemical production but also for design of new sensors, electronics, medicine, and, especially, for fuel cells [15].

The most desirable structures for nanoporous materials are columnar or cubic phases. The use of columnar phases is more developed for conducting proposes but the control of channels orientation in such phases can be an issue. Thus, for optimizing the ion transport across the membrane in the columnar phase, one has to induce vertical or, the so-called homeotropic, orientation of the columns [16]. This is a nontrivial task because, in many instances, such orientation is not thermodynamically stable. The bicontinuous cubic phases do not require macroscopic orientation because the channels in such structures are running along all three directions in space. The cubic phases can be described in terms of triply periodic minimal surfaces (TPMSs). The TPMSs represent nonintersecting surfaces with three-dimensional (3D) periodicity and vanishing mean curvature $H$, where $H=\frac{1}{2}\left(\kappa_{1}+\kappa_{2}\right)$, and $\kappa_{1}$ and $\kappa_{2}$ stand for the principal curvatures at the point [17]. Such continuous surface separates the space into two interwoven nets of channels known as bicontinuous structure. The most commonly known TPMSs are the Schwarz primitive (P), Schwarz diamond (D), and Schoen gyroid $(\mathrm{G})$ surface structures.

The TPMSs are intriguing due to their ability to exhibit unique physical properties. For example, such surfaces are used to model different crystalline structures in both natural and synthetic systems. Examples of these structures include LLCs and TLCs [18,19], block copolymer (BCP) self-assemblies [2,20-22] and organic zeolites [23]. It is noteworthy that one important structural difference between the LC and BCP systems consists in the values of the unit cell parameter, which ranges between ca. 10 and $100 \mathrm{~nm}$ for BCP-based systems [24], while it can rarely exceed several nanometers for LC systems [25]. In some instances, it is interesting to make the characteristic distances of both systems closer to each other, i.e., by increasing the sizes of the LC systems and/or by bringing down the dimensions of phases generated by the BCPs in order to make them interesting for the developing areas of nanotechnologies and nanopatterning [26].

The wedge-shaped molecules can be considered as building units for design of zeolite-like materials that can be excellent candidates for fabrication of nanoporous membranes [27-30]. It is already established that these compounds are able to self-organize in complex morphologies pertinent to the cubic bicontinuous phases, such as diamond, gyroid, and primitive cubic phases. The family of bicontinuous cubic phases can be considered as an organic equivalent of zeolites due to their developed network of welldefined nanochannels. Among these structures, gyroid bicontinuous phases are considered as being more accessible for practical applications because of self-supporting frameworks with better mechanical strength, ensuring open-cell character, high and uniform porosity, and large and predictable specific surface area. However, the gyroid phases formation mechanism and corresponding functionality are still not sufficiently studied. This is in part accounted for by experimental difficulties in studying the structure formation process in different environments with the necessity to perform in-situ structural characterizations. Therefore, several scientific and technical challenges have still to be taken to get a full understanding of the corresponding structure-property relationships, as well as details of the gyroid phase formation process.

For several years, our group has been involved in studies of self-assembly processes in various wedge-shaped compounds based on sulfonic acid. We have previously shown that, during heating or UV-irradiation (for the systems with light-sensitive groups), these systems form a variety of LC morphologies, such as smectic, columnar, and cubic (double diamond and gyroid). Swelling of thin films in solvent vapors in some cases leads to development of ion channel networks, with the lattice parameter becoming comparable to the one in the BCP systems [31-35]. Earlier, we have reported on formation of wellorganized cubic phase in the films of wedge-shaped salts with linear alkyl chains during swelling in methanol. Surprisingly, this mesophase was found to be rather stable in a wide 
temperature range, and, upon preparation, the methanol solvent can be replaced by water in ion channels, which makes these systems interesting for future applications [33-35]. Synchrotron Grazing-Incidence X-ray scattering (GISAXS) experiments in combination with computer simulation reveal development of water channels of ca. $2 \mathrm{~nm}$ in diameter (cf. Figure 1), which makes them a promising candidate for fabrication of mechanically stable membranes with excellent proton conductivity.

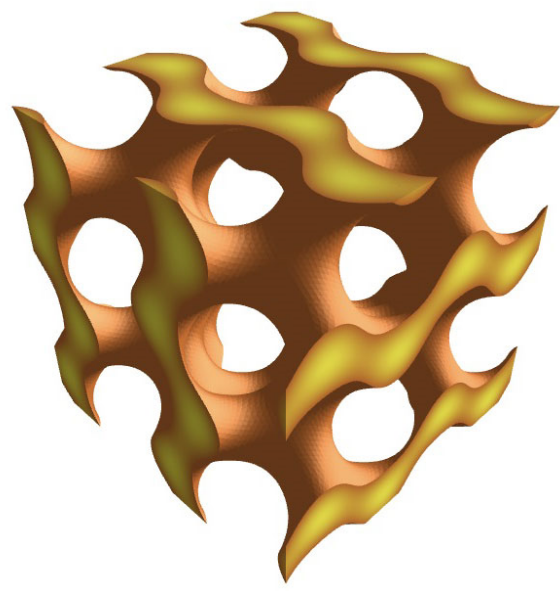

(a)

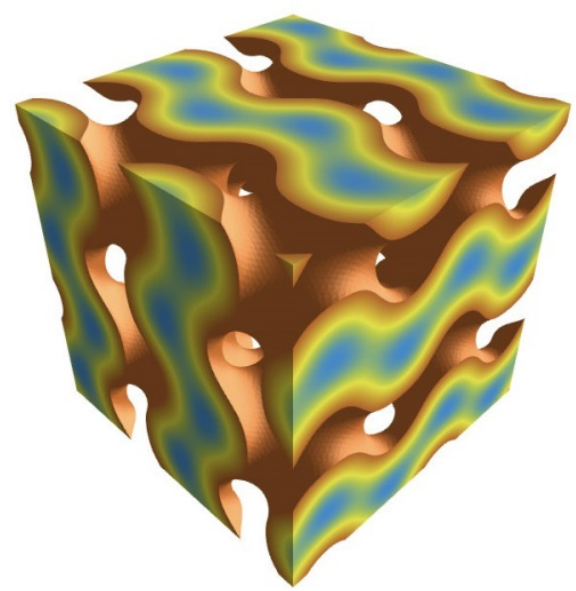

(b)

Figure 1. Three-dimensional model of a bicontinuous double gyroid phase of a wedge-shaped amphiphile in a dry (a) and swollen (b) state. Yellow chocolate color indicates the hydrophilic part of amphiphilic molecules, while the blue color denotes the water inside the molecular channels. The structure in (b) contains water fraction 33 wt.\%.

In the present study, we address the development of ion-channel network during swelling in humid atmosphere by in-situ monitoring the structural evolution and topography of thin films of a wedge-shaped amphiphile.

\section{Materials and Methods}

The synthesis of pyridinium salt of $4^{\prime}$-[3", 4 ", 5 "'-tris-(octyloxy)benzoyloxy]azobenzene4-sulfonic acid (C8AzoPyr) was described in previous reports [31,36]. The chemical structure of C8AzoPyr is given in Scheme 1.

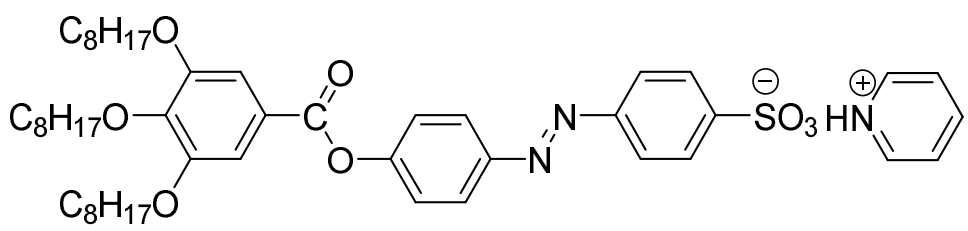

Scheme 1. The chemical structure of the studied wedge-shaped sulfonate molecule C8AzoPyr.

Differential Scanning Calorimetry (DSC) measurements were carried out using a Netzsch DSC 204 unit. Samples (typical weight of $5 \mathrm{mg}$ ) were enclosed in standard Netzsch $25 \mu \mathrm{L}$ aluminum crucibles. Heating and cooling rate was $10^{\circ} \mathrm{C} / \mathrm{min}$.

Grazing-Incidence Small- and Wide-Angle X-ray scattering (GISAXS-GIWAXS) measurements were performed at the BM26 and ID10 beamlines of the European Synchrotron Radiation Facility (ESRF) in Grenoble (France) using a custom-designed environmental chamber [34,35].

The energy of X-ray photons was $12 \mathrm{keV}$. The s-axis $(|\mathbf{s}|=2 \sin \theta / \lambda$, where $\theta$ is the Bragg angle, $\lambda$ is the wavelength, and $|\mathbf{s}|$ is the norm of the $\mathbf{s}$-vector) was calibrated using several diffraction orders of silver behenate. X-ray patterns were recorded using a $2 \mathrm{D}$ 
Pilatus $1 \mathrm{M}$ camera. The $\mathrm{X}$-ray data analysis, including background subtraction and radial integrations of the 2D patterns were accomplished using home-built routines designed within the IgorPro software package (Version 6.37, Wavemetrics Ltd., Portland, OR, USA). For the GISAXS experiments, thin films of C8AzoPyr were prepared from a chloroform solution $(20 \mathrm{mg} / \mathrm{mL})$ by spin-coating $(500 \mathrm{rpm} / \mathrm{min})$ on a silicon wafer substrate. The phase composition of the thin films at different temperatures was addressed by in-situ heating and cooling of the samples in temperature range from $-50^{\circ}$ to $100^{\circ} \mathrm{C}$. The change in the phase structure during the water uptake process in thin films was also monitored using in-situ control of relative humidity $(\mathrm{RH})$ and temperature.

A specially designed environmental cell was used for in-situ studies of the structural evolution in thin films (Figure 2). The compactness of experimental cell makes it possible to use it on the synchrotron beamlines, i.e., in the sample chambers of a diffractometer or spectrophotometer, and can be also combined with an optical microscope. The experimental cell allows one to study thin films by the GIWAXS/GISAXS methods with simultaneous control of external factors, such as UV radiation, temperature, and atmosphere of solvent vapors. The experimental cell includes the following components: a sealed chamber, consisting of a heating element, a lid to create the desired atmosphere over the sample, a frame for mounting, and a temperature and humidity control system.

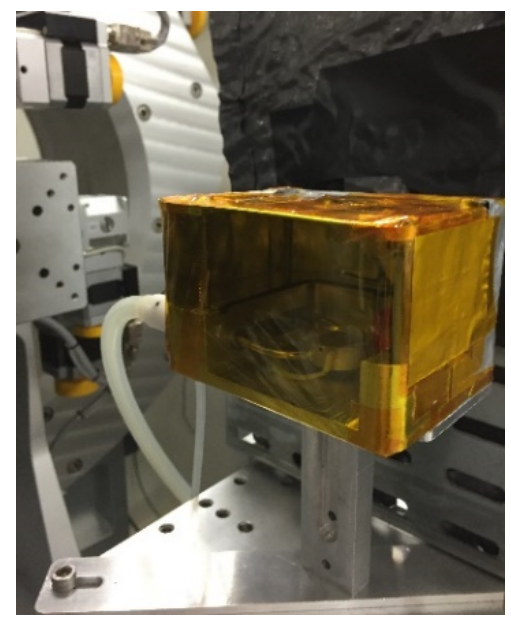

(a)

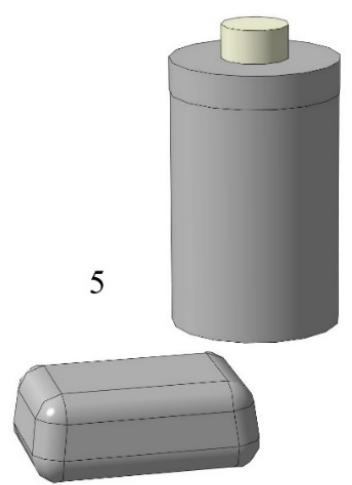

(b)

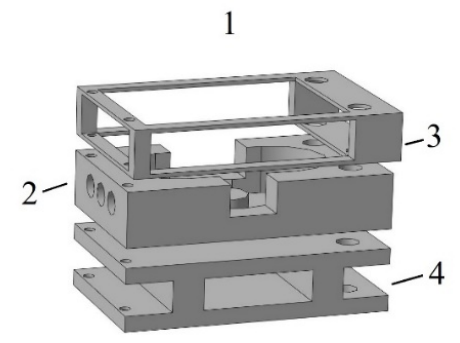

Figure 2. (a): Environmental cell for in-situ control of the atmosphere and temperature installed at BM26 beamline of ESRF; (b): schematics of the environmental cell components: (1)-sealed chamber, composed of a heating element (2), lid (3) to create controlled atmosphere around the sample, frame (4) for mounting, temperature and humidity control system. The latter consists of a dewar with liquid nitrogen (depicted as a cylinder) and a control panel/computer (depicted as a box) (5).

AFM imaging was performed in Tapping Mode using a Cypher S Asylum Research Atomic Force Microscope. In the experiments, Oxford Instruments AC240TS medium soft silicon cantilevers (Abingdon, UK) with a resonance frequency $67 \mathrm{kHz}$ and spring constant $1.82 \mathrm{~N} / \mathrm{m}$ were used. The spring constant of the cantilevers was measured by the thermal noise method. To conduct measurements at variable humidity, a special portable air humidity control system was employed. The system shown on Scheme 2a consists of a computer connected to a controller and aeraulic circuit of humidity regulator (Scheme $2 b$ ). The main controller regulates the flow of the compressed air through the system. In order to create humidity-controlled air, dry and humid air are mixed in the desired proportions with the help of Mass Flow Controllers (MFC) for different flow ranges connected to the main controller. This air is then redirected through the Atomic Force Microscope Cypher $S$ Asylum Research equipped with humidity sensor that allows to maintain the required humidity value during the experiment. The AFM images were analyzed using the open source software Gwyddion (Version 2.58). 


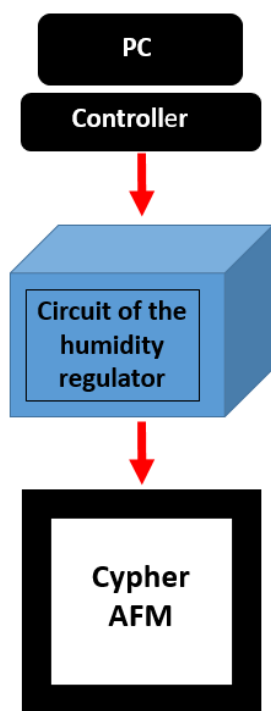

(a)

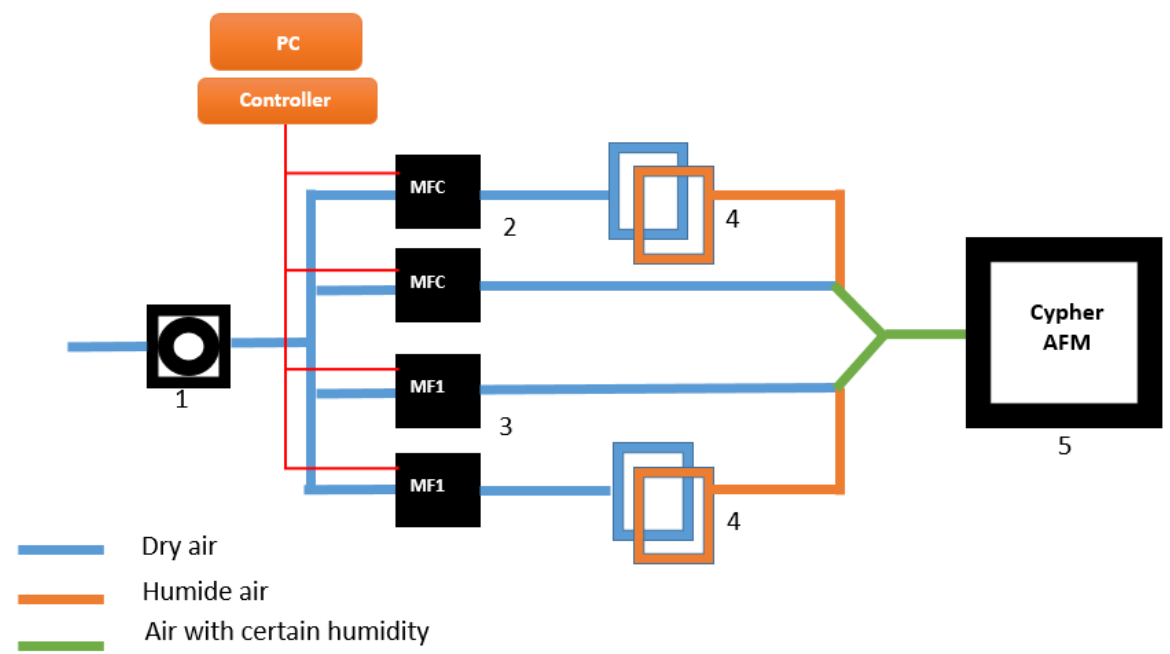

(b)

Scheme 2. (a): Scheme of portable air humidity control system; (b): aeraulic circuit of the humidity regulator connected to controller and PC: 1-manometer, 2-Mass Flow Controller with flow range of 10,000 sccm, 3-Mass Flow Controller MF-1 with flow range of $100 \mathrm{sccm}$, 4-laboratory flask with water, 5-Atomic Force Microscope Cypher S Asylum Research.

\section{Results}

The thermal behavior of C8AzoPyr was assessed by DSC. The DSC trace corresponding to the first heating exhibits two endothermic peaks (Figure 3, black line). The first peak with onset at $72{ }^{\circ} \mathrm{C}$, and enthalpy $0.7 \mathrm{~J} / \mathrm{g}$ is associated with a solid-solid state transition; the second intense endothermic peak with onset at $96^{\circ} \mathrm{C}$, and enthalpy $26 \mathrm{~J} / \mathrm{g}$ can be attributed to melting of a crystalline structure. Interestingly, the second heating curve does not exhibit endothermic peaks anymore (Figure 3, red line). In our earlier report, crystallization of C8AzoPyr from a LC phase during annealing at room temperature was explained by a process of local ordering of linear octyl chains [35]. Figure S1a shows FTIR spectrum of $\mathrm{C} 8 \mathrm{AzoPyr}$ after a long storage at room temperature. In the magnified region from 3000 to $2800 \mathrm{~cm}^{-1}$, one can see that conformation-sensitive anti-symmetric and symmetric stretching vibration modes of $\mathrm{CH}_{2}$ group are positioned at 2921 and $2851 \mathrm{~cm}^{-1}$, respectively (cf. Figure S1b). These values are close to those characteristic of all-trans conformation of n-alkanes-2920 and $2850 \mathrm{~cm}^{-1}$, respectively [37]. Consequently, in the crystalline state, $\mathrm{C} 8 \mathrm{AzoPyr}$ possesses ordered octyl chains in a quasi-extended conformation. Above the melting point, the structure reorganizes to a columnar hexagonal LC phase with disordered alkyl chains (Figure S2a,b). Since the second heating performed after 3 min at room temperature does not reveal any transitions, the LC-to-crystal phase transition at room temperature is likely to be a slow process (Figure S2c,d). This gives one the time to manipulate the structure while in the mobile LC state by varying the external factors. In contrast, in the crystalline state, a rigid framework of side chains prevents any structural evolution in the atmosphere of water vapors or in vapors of organic solvents [35].

Figure 4a displays GISAXS pattern after long storage at room temperature. The ordered phase was indexed to a columnar monoclinic unit cell $\left(\mathrm{Col}_{\mathrm{mon}}\right)$, which has the following parameters: $\mathrm{a}=58.9 \AA \mathrm{A}, \mathrm{b}=50.0 \AA, \gamma=61^{\circ}$. However, in the LC state with liquid-like side chains which is generated upon cooling from $100{ }^{\circ} \mathrm{C}$, the structure of C8AzoPyr is more sensitive to vapors of polar solvents, such as water or alcohols, due to their more effective diffusion through the amorphous alkyl periphery of the molecular wedges. Thus, swelling in methanol atmosphere for $2 \mathrm{~h}$ results in formation of a layer-like lamellar structure with parameter $\mathrm{a}_{\mathrm{lam}}=51.2 \AA$ (Figure $4 \mathrm{~b}$ ). The corresponding indexed 1D-reduced diffractograms are presented in Figure S3. 
Earlier, we demonstrated that during slow cooling from $100{ }^{\circ} \mathrm{C}$ in a methanol atmosphere the high-temperature columnar hexagonal structure transforms to a stable cubic double gyroid structure [35]. It is noteworthy that the normal to the film surface becomes parallel to the 211 reciprocal space direction, as was previously noted for another wedgeshaped mesogen [31]. After several months annealing at ambient conditions, the gyroid structure not only persists but even shows a definite improvement of its organization. POM images of the dry film (not shown here) confirm the absence of birefringence which is typical of cubic phases. The diffraction pattern of C8AzoPyr thin film demonstrates a set of peaks with d-spacings ratio of $\sqrt{6}: \sqrt{8}$, which is characteristic of the Cub gyr phase (symmetry $I a \overline{3} d$ ). The corresponding lattice parameter agyr is $117 \AA$ (Figure $4 \mathrm{c}$ ). The summary of the extinction rules for the different bicontinuous phases can be found in ref. [38].

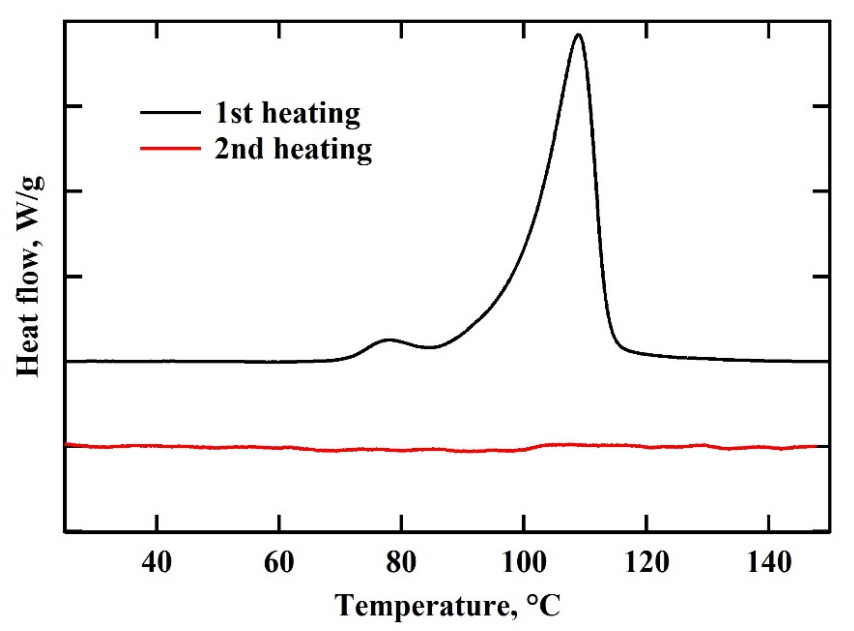

Figure 3. DSC curves: first (black) and second (red) heating traces of bulk C8AzoPyr after long annealing at room temperature.

The presence of bicontinuous network of opened hydrophilic channels makes the film capable of efficiently swelling in a humid atmosphere. A 2D GISAXS pattern of the thin film with the Cubgyr phase corresponding to the sample kept for two days in saturated water vapors reveals a significant increase of the lattice parameter agyr to $138 \AA$. This is an indication of formation of bicontinuous networks of water nanochannels (Figure 4d). The average radii of water channels in gyroid phase in the as-prepared and swollen film is found to be $8 \pm 1$ and $10 \pm 2 \AA$, respectively [35]. Consequently, formation of Cub $b_{\text {gyr }}$ phase stabilized by ordered alkyl chains demonstrates the outstanding ability of the system to absorb water without destruction of supramolecular organization with long-range order. The swelling process of C8AzoPyr was further studied by in-situ AFM.

Figure 5a shows a topographic image of a C8AzoPyr thin film exposed to relative humidity of $42 \%$. The film clearly exhibits a terrace-like structure that can be quantitatively analyzed using height cross-sections, as shown in Figure 5b. In this case, the cross-section traced along the black solid line in Figure 5a reveals terraces with an average height of $47.5 \AA$. Since the vertical direction of the film corresponds to the 211 reciprocal space vector of the double gyroid phase according to GISAXS, it is logical to assume that the corresponding step height should give the reticular distance of the (211) planes of $\frac{a}{\sqrt{6}}$. This allows computing the lattice parameter $\mathrm{a}_{\text {gyr }}$ of $116 \AA$.

However, if one wants to analyze the gyroid structure with more scrutiny, it would be necessary to image the film surface with higher resolution. An example of such measurement is given in Figure 6, where AFM phase images of C8Pyr thin films at different $\mathrm{RH}$ values are shown. Despite some noise present in the images (the images after filtering are shown in the insets), one can recognize the unique pattern of the 211 plane of the double gyroid phase (see, e.g., Reference [39]). The observed morphological features of the 211 plane can be viewed relative to the $1 \overline{11}$ and $01 \overline{1}$ reciprocal space vectors that direct 
along and perpendicular to the characteristic "knitting" features of the plane, respectively. The analysis in the Fourier space given in the right column of Figure 6 allows visualizing the characteristic in-plane periodicities. Thus, the image taken in the dry $(\mathrm{RH}=20 \%)$ state of the film reveals the fundamental periodicity along the $01 \overline{1}$ direction of $0.057 \mathrm{~nm}^{-1}$, which is equivalent to the distance of $17.5 \mathrm{~nm}$ in direct space. The periodicity in the vertical direction of $0.09 \mathrm{~nm}^{-1}$ provides the corresponding direct-space repeat of $11.1 \mathrm{~nm}$. Taking into account the expressions for both distances given in ref. [39], one obtains the lattice parameter of the gyroid phase to be 12.4 and $12.8 \mathrm{~nm}$, respectively. These values agree relatively well with the corresponding SAXS unit cell parameter, which validates such AFM-based analysis. A small difference (of ca. $3 \%$ ) in the values computed for the horizontal and vertical directions can be accounted for by a small drift of the piezo along the slow scan direction.

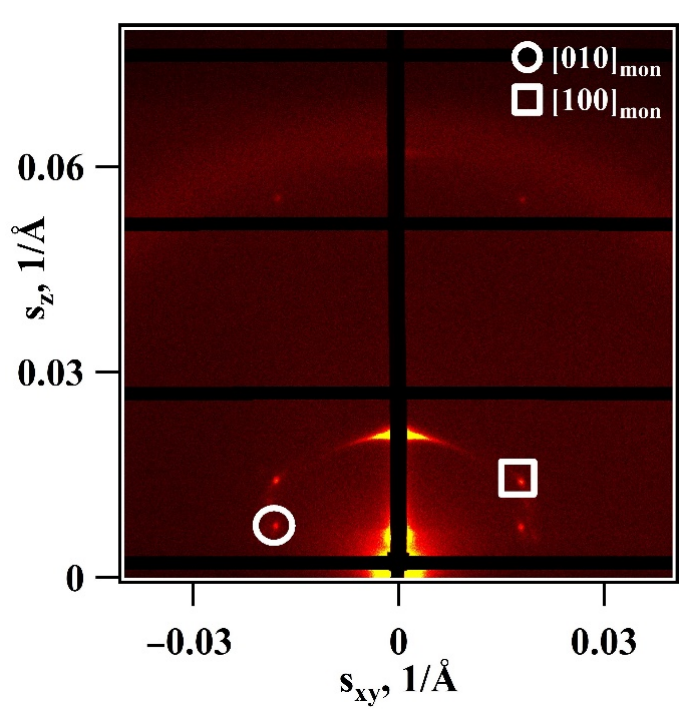

(a)

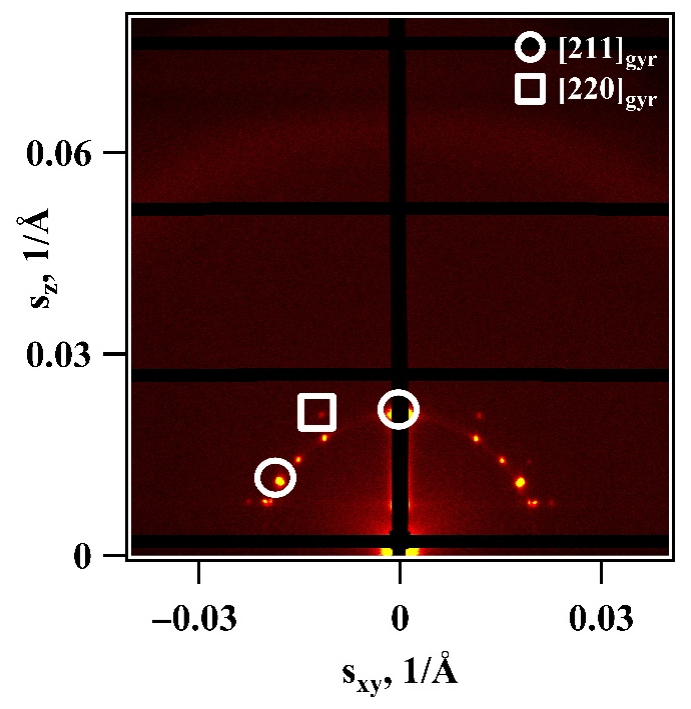

(c)

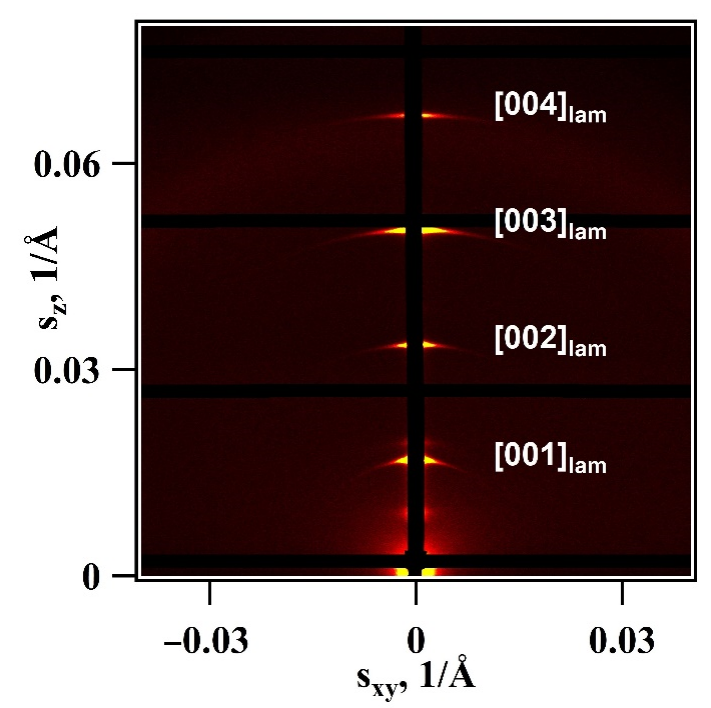

(b)

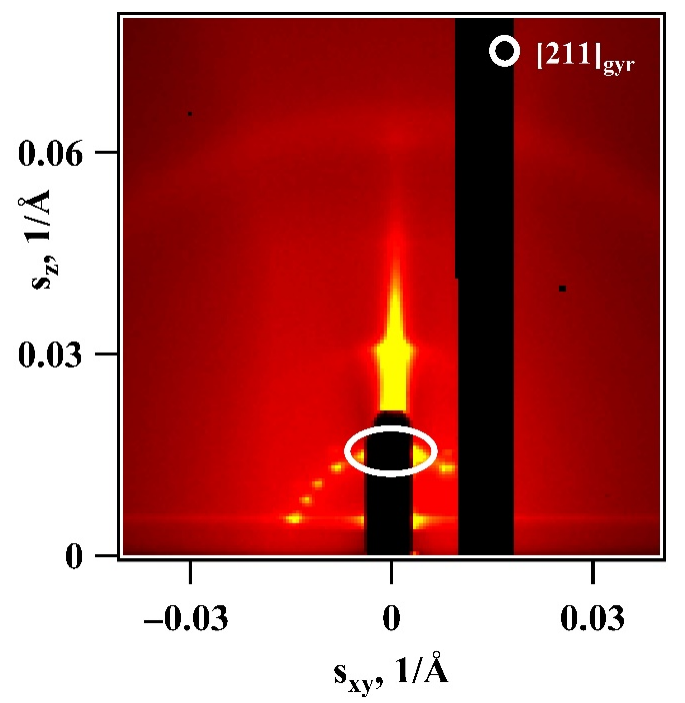

(d)

Figure 4. Two-dimensional GISAXS pattern of a dry C8AzoPyr thin film. (a) Colmon phase formed after long annealing at room temperature; (b) lamellar phase formed upon swelling in methanol vapors for two hours at room temperature; (c) Cub gyr phase generated after long-term annealing at ambient conditions; (d) Cubgyr phase swollen in water vapors for two days at room temperature. 


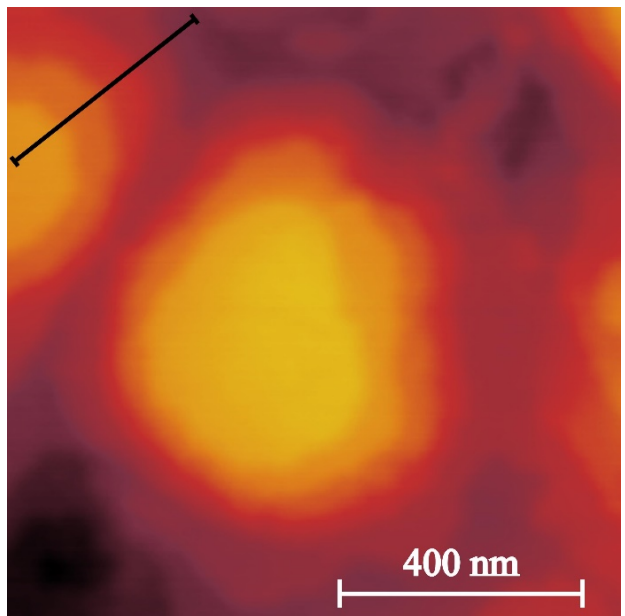

(a)

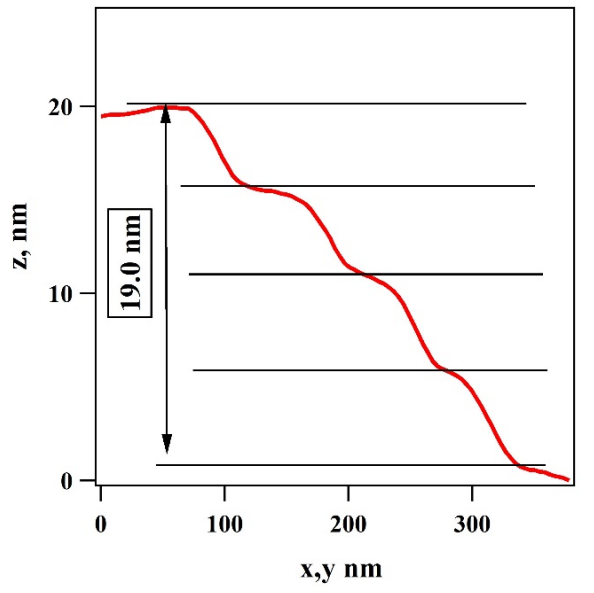

(b)

Figure 5. AFM image of a C8AzoPyr thin film: (a) height trace of the film surface at $\mathrm{RH}=42 \%$; (b) height cross-section traced along the black solid line in (a) showing quantized terraces.

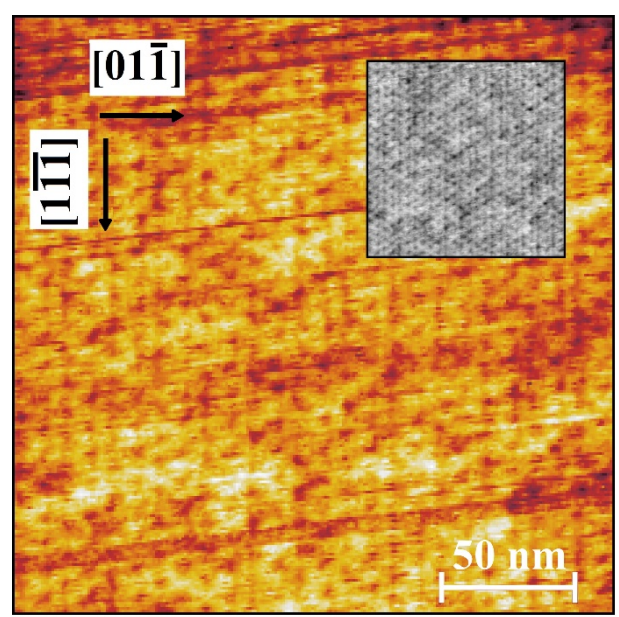

(a)

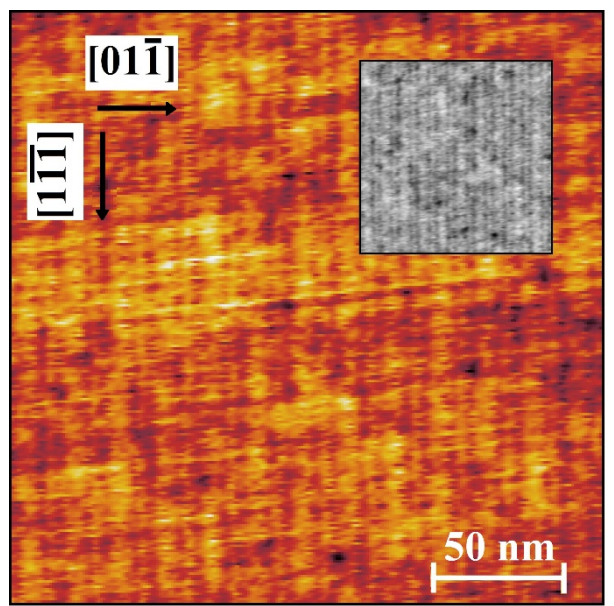

(c)

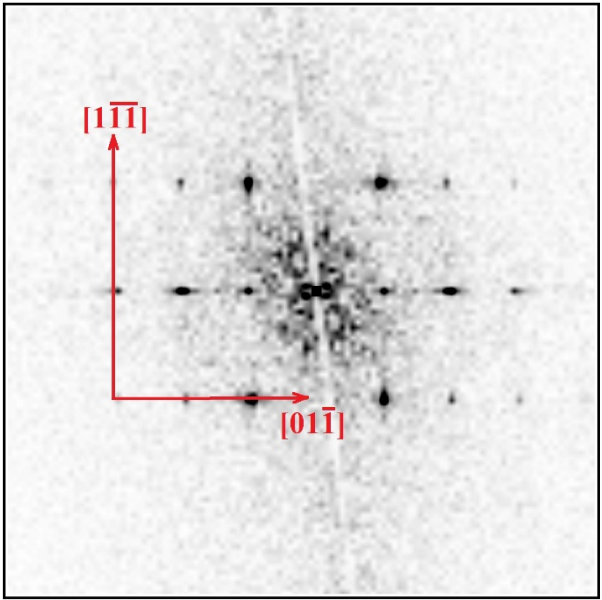

(b)

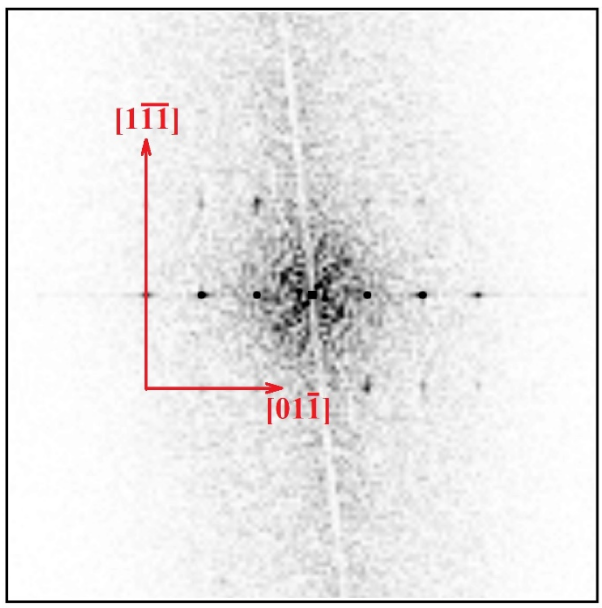

(d)

Figure 6. AFM images of the C8AzoPyr sample: (a) phase image and (b) FT pattern of the dry film $(\mathrm{RH}=20 \%)$; (c) phase image and (d) FT pattern of the swollen film $(\mathrm{RH}=100 \%)$. The insets show the corresponding AFM images after filtering in the Fourier space. 
The described reciprocal-space analysis makes it possible to quantitatively compare the AFM phase images of the gyroid phase taken at different values of RH. Indeed, the two images in Figure 6 can be confronted based on the positions of the characteristic peaks in the Fourier space. The computation of the unit cell parameter for the image taken at 100\% RH gives the value of $13.5 \mathrm{~nm}$, which is noticeably larger than the corresponding value in the dry state and is also close to the SAXS unit cell parameter measured for the long-term annealing of the sample in the saturated water vapors. The presented AFM results confirm the unusually high stability of the Cub gyr phase in swelling-drying cycles.

\section{Discussion}

The present work provides an example of a wedge-shaped C8AzoPyr amphiphile bearing promise for applications in ion-selective membranes for separation, catalysis, and energy conversion. To optimize the ion transport, one has to prepare the $\mathrm{C} 8 \mathrm{AzoPyr}$ membranes in a bicontinuous cubic phase, such as the double gyroid $I a \overline{3} d$. The experiments reveal a very rich polymorphic behavior of C8AzoPyr both in LLC and TLC phases, which makes any search for the desired cubic phase exclusively with ex-situ techniques inefficient. Here, we show that such search employing a combination of environmental in-situ GISAXSGIWAXS and AFM experiments is much more productive as one can easily operate the external factors, such as solvent vapors and temperature, while continuously monitoring the film structure.

An interesting particularity of the studied system is that the gyroid phase can be locked in the film and persist even in its fully dry state. This is completely unusual for the LLC phases for which the gyroid phase exists only in a certain range of solvent fractions. Such locking the gyroid phase is accounted for by ordering of the octyl side chains that keep the gyroid phase stable in the course of repeated swelling-drying cycles. Such metastable state can be erased only if one melts the side-chains' locks at high temperature. However, as long as the side chains keep their local ordering, the formed gyroid phase will persist, and the material will preserve its functionality. Therefore, it is shown that, by combining in-situ structure monitoring with reciprocal- and direct-space experimental techniques and optimized molecular architecture, one can design the materials with enhanced stability of the bicontinuous structure.

\section{Conclusions}

Using environmental in-situ grazing-incidence $\mathrm{X}$-ray diffraction and atomic force microscopy, the phase behavior of thin films of a wedge-shaped mesogen in dry and swollen state was investigated. The studied samples show the presence of unusually stable bicontinuous cubic double gyroid phase, whereas, in the swollen thin films, they reveal formation of swollen water channels. The obtained results can help understanding the structure formation in supramolecular systems at different hierarchical levels and, consequently, contribute to the development of new approaches in fabrication of selforganized films with the desired morphology.

Supplementary Materials: The following are available online at https:/ /www.mdpi.com/article/10 .3390/ma14112892/s1, Figure S1: (a) FTIR spectrum of C8AzoPyr powder after long-term storage at room temperature; (b) magnified region of (a) with anti-symmetric and symmetric stretching vibration modes of $\mathrm{CH} 2$ groups, Figure S2: 2D GISAXS patterns of a dry C8AzoPyr thin film recorded during first heating from $25^{\circ} \mathrm{C}$ at $80^{\circ} \mathrm{C}$ (a) and at $100{ }^{\circ} \mathrm{C}$ (b), and during second heating from $25^{\circ} \mathrm{C}$ at $80^{\circ} \mathrm{C}$ (c) and at $100{ }^{\circ} \mathrm{C}(\mathrm{d})$, Figure S3: 1D-reduced GISAXS pattern of a dry C8AzoPyr thin film. (a) Columnar monoclinic (Colmon) phase formed after long-term annealing at room temperature; (b) lamellar phase (lam) formed upon swelling in methanol vapors for two hours at room temperature; (c) double gyroid phase (Cubgyr) generated after long-term annealing at ambient conditions; (d) Cubgyr phase swollen in water vapors for two days at room temperature. 
Author Contributions: Conceptualization, K.N.G.; Data curation, D.S.K.; Formal analysis, D.V.A.; Investigation, A.F.A.; Methodology, D.V.V., A.F.A., X.Z., D.P.; Supervision, D.A.I.; writing-original draft preparation, K.N.G.; Writing-original draft, D.V.A., D.A.I.; Writing-review and editing, D.A.I. All authors have read and agreed to the published version of the manuscript.

Funding: This research was funded by the Ministry of Science and High Education of the Russian Federation (contract No 05.605.21.0188 from 3 December 2019 (RFMEFI60519X0188)).

Institutional Review Board Statement: Not applicable.

Informed Consent Statement: Not applicable.

Data Availability Statement: Data available on request due to restrictions e.g., privacy or ethical. The data presented in this study are available on request from the corresponding author. The data are not publicly available due to organization policy.

Acknowledgments: We thank ESRF for beamtime at BM26, ESRF's Partnership for Soft Condensed Matter (PSCM) for support and A.Panzarella and M.Capron for help in AFM experiments and A.Rychkov for the sketch of the GISAXS environmental cell.

Conflicts of Interest: The authors declare no conflict of interest. The funders had no role in the design of the study; in the collection, analyses, or interpretation of data; in the writing of the manuscript, or in the decision to publish the results.

\section{References}

1. Petsko, G.A.; Ringe, D. Protein Structure and Function; New Science Press, Ltd.: London, UK, 2004.

2. Mai, Y.; Eisenberg, A. Self-assembly of block copolymers. Chem. Soc. Rev. 2012, 41, 5969-5985. [CrossRef]

3. Yashima, E.; Ousaka, N.; Taura, D.; Shimomura, K.; Ikai, T.; Maeda, K. Supramolecular helical systems: Helical assemblies of small molecules, foldamers, and polymers with chiral amplification and their functions. Chem. Rev. 2016, 116, 13752-13990. [CrossRef] [PubMed]

4. Borisch, K.; Diele, S.; Göring, P.; Kresse, H.; Tschierske, C. Tailoring thermotropic cubic mesophases: Amphiphilic polyhydroxy derivatives. J. Mater. Chem. 1998, 8, 529-543. [CrossRef]

5. Borisch, K.; Tschierske, C.; Göring, P.; Diele, S. Molecular design of thermotropic liquid crystalline polyhydroxy amphiphiles forming type 1 columnar and cubic mesophases. Langmuir 2000, 16, 6701-6708. [CrossRef]

6. Fong, C.; Le, T.; Drummond, C.J. Lyotropic liquid crystal engineering-ordered nanostructured small molecule amphiphile self-assembly materials by design. Chem. Soc. Rev. 2012, 41, 1297-1322. [CrossRef]

7. Mezzenga, R.; Seddon, J.M.; Drummond, C.J.; Boyd, B.J.; Schröder-Turk, G.E.; Sagalowicz, L. Nature-Inspired Design and Application of Lipidic Lyotropic Liquid Crystals. Adv. Mater. 2019, 31, 1900818. [CrossRef] [PubMed]

8. Zhai, J.; Fong, C.; Tran, N.; Drummond, C.J. Non-Lamellar Lyotropic Liquid Crystalline Lipid Nanoparticles for the Next Generation of Nanomedicine. ACS Nano 2019, 13, 6178-6206. [CrossRef]

9. Barriga, H.M.G.; Holme, M.N.; Stevens, M.M. Cubosomes: The Next Generation of Smart Lipid Nanoparticles? Angew. Chem. Int. Ed. 2019, 58, 2958-2978. [CrossRef]

10. Caffrey, M. A comprehensive review of the lipid cubic phase or in meso method for crystallizing membrane and soluble proteins and complexes. Struct. Biol. Commun. 2015, 71, 3-18.

11. Zabara, A.; Meikle, T.G.; Newman, J.; Peat, T.S.; Conn, C.E.; Drummond, C.J. The nanoscience behind the art of in-meso crystallization of membrane proteins. Nanoscale 2017, 9, 754-763. [CrossRef]

12. Reppe, T.; Poppe, S.; Tschierske, C. Controlling Mirror Symmetry Breaking and Network Formation in Liquid Crystalline Cubic, Isotropic Liquid and Crystalline Phases of Benzil-Based Polycatenars. Chem. Eur. J. 2020, 26, 16066-16079. [CrossRef] [PubMed]

13. Zhang, L.; Jaroniec, M. Strategies for development of nanoporous materials with 2D building units. Chem. Soc. Rev. 2020, 49, 6039-6055. [CrossRef] [PubMed]

14. Wang, Z.; Yu, J.; Xu, R. Needs and trends in rational synthesis of zeolitic materials. Chem. Soc. Rev. 2012, 41, 1729-1741. [CrossRef] [PubMed]

15. Xiang, Z.; Cao, D.; Dai, L. Well-defined two dimensional covalent organic polymers: Rational design, controlled syntheses, and potential applications. Polym. Chem. 2015, 6, 1896-1911. [CrossRef]

16. Gearba, R.I.; Anokhin, D.V.; Bondar, A.I.; Bras, W.; Lehmann, M.; Ivanov, D.A. Homeotropic Alignment of Columnar Liquid Crystals in Open Films by Means of Surface Nano-Patterning. Adv. Mater. 2007, 19, 815-820. [CrossRef]

17. Hyde, S.; Blum, Z.; Landh, T.; Lidin, S.; Ninham, B.W.; Andersson, S.; Larsson, K. The Language of Shape: The Role of Curvature in Condensed Matter: Physics, Chemistry and Biology; Elsevier: Amsterdam, The Netherlands, 1996.

18. Fontell, K. Cubic phases in surfactant and surfactant-like lipid systems. Colloid Polym. Sci. 1990, 268, 264-285. [CrossRef]

19. Seddon, J.M. An inverse face-centered cubic phase formed by diacylglycerol-phosphatidylcholine mixtures. Biochemistry 1990, 29, 7997-8002. [CrossRef] 
20. Orilall, M.C.; Wiesner, U. Block copolymer based composition and morphology control in nanostructured hybrid materials for energy conversion and storage: Solar cells, batteries, and fuel cells. Chem. Soc. Rev. 2011, 40, 520-535. [CrossRef]

21. Cochran, E.W.; Garcia-Cervera, C.J.; Fredrickson, G.H. Stability of the gyroid phase in diblock copolymers at strong segregation. Macromolecules 2006, 39, 2449-2451. [CrossRef]

22. Stefik, M.; Guldin, S.; Vignolini, S.; Wiesner, U.; Steiner, U. Block copolymer self-assembly for nanophotonics. Chem. Soc. Rev. 2015, 44, 5076-5091. [CrossRef]

23. Sun, J.; Bonneau, C.; Cantín, A.; Corma, A.; Díaz-Cabañas, M.J.; Moliner, M.; Zhang, D.; Li, M.; Zou, X. The ITQ-37 mesoporous chiral zeolite. Nature 2009, 458, 1154-1157. [CrossRef] [PubMed]

24. Meuler, A.J.; Hillmyer, M.A.; Bates, F.S. Ordered network mesostructures in block polymer materials. Macromolecules 2009, 42, 7221-7250. [CrossRef]

25. Beginn, U. Thermotropic columnar mesophases from $\mathrm{N}-\mathrm{H} \cdots \mathrm{O}$, and $\mathrm{N} \cdots \mathrm{H}-\mathrm{O}$ hydrogen bond supramolecular mesogenes. Prog. Polym. Sci. 2003, 28, 1049-1105. [CrossRef]

26. Miskaki, C.; Moutsios, I.; Manesi, G.-M.; Artopoiadis, K.; Chang, C.-Y.; Bersenev, E.A.; Moschovas, D.; Ivanov, D.A.; Ho, R.-M.; Avgeropoulos, A. Self-Assembly of Low-Molecular-Weight Asymmetric Linear Triblock Terpolymers: How Low Can We Go? Molecules 2020, 25, 5527. [CrossRef] [PubMed]

27. Zhu, X.; Beginn, U.; Möller, M.; Gearba, R.I.; Anokhin, D.V.; Ivanov, D.A. Self-Organization of polybases neutralized with mesogenic wedge-shaped sulfonic acid molecules: An approach toward supramolecular cylinders. Am. Chem. Soc. 2006, 128, 16928-16937. [CrossRef]

28. Hernandez Rueda, J.J.; Zhang, H.; Rosenthal, M.; Möller, M.; Zhu, X.; Ivanov, D.A. Polymerizable wedge-shaped ionic liquid crystals for fabrication of ion-conducting membranes: Impact of the counterion on the phase structure and conductivity. Eur. Polym. J. 2016, 81, 674-685. [CrossRef]

29. Chen, Y.; Lingwood, M.D.; Goswami, M.; Kidd, B.E.; Hernandez, J.J.; Rosenthal, M.; Ivanov, D.A.; Perlich, J.; Zhang, H.; Zhu, X.; et al. Humidity-Modulated Phase Control and Nanoscopic Transport in Supramolecular Assemblies. J. Phys. Chem. B 2014, 118, $3207-3217$. [CrossRef] [PubMed]

30. Hernandez, J.J.; Zhang, H.; Chen, Y.; Rosenthal, M.; Lingwood, M.D.; Goswami, M.; Zhu, X.; Moeller, M.; Madsen, L.A.; Ivanov, D.A. Bottom-Up Fabrication of Nanostructured Bicontinuous and Hexagonal Ion-Conducting Polymer Membranes. Macromolecules 2017, 50, 5392-5401. [CrossRef]

31. Zhang, H.; Li, L.; Moller, M.; Zhu, X.; Hernandez Rueda, J.J.; Rosenthal, M.; Ivanov, D.A. From channel-forming ionic liquid crystals exhibiting humidity-induced phase transitions to nanostructured ion-conducting polymer membranes. Adv. Mater. 2013, 25, 3543-3548. [CrossRef]

32. Grafskaia, K.N.; Hernandz Rueda, J.J.; Zhu, X.; Nekipelov, V.M.; Anokhin, D.V.; Moeller, M.; Ivanov, D.A. Designing the topology of ion nano-channels in the mesophases of amphiphilic wedge-shaped molecules. Phys. Chem. Chem. Phys. 2015, 17, 30240-30247. [CrossRef]

33. Grafskaia, K.N.; Anokhin, D.V.; Hernandez Rueda, J.J.; Ivanov, D.A. In situ studies of molecular self-assembling during the formation of ion-conducting membranes for fuel cells. Appl. Mech. Mater. 2015, 792, 623-628. [CrossRef]

34. Grafskaia, K.; Zimka, B.; Zhu, X.; Anokhin, D.; Ivanov, D. Engineering of ion channels topology in self-assembled wedge-shaped amphiphiles by combination of temperature and solvent vapor treatment. AIP Conf. Proc. 2016, 1748, 040009.

35. Grafskaia, K.N.; Anokhin, D.V.; Zimka, B.I.; Izdelieva, I.A.; Zhu, X.; Ivanov, D.A. An “on-off” switchable cubic phase with exceptional thermal stability and water sorption capacity. Chem. Commun. 2017, 53, 13217-13220. [CrossRef]

36. Zhu, X.; Tartsch, B.; Beginn, U.; Möller, M. Wedge-Shaped Molecules with a Sulfonate Group at the Tip-A New Class of Self-Assembling Amphiphiles. Chem. Eur. J. 2004, 10, 3871-3878. [CrossRef]

37. Snyder, R.G.; Strauss, H.L.; Elliger, C.A. Carbon-hydrogen Stretching Modes and the Structure of n-Alkyl Chains. 1. Long Disordered Chains. J. Phys. Chem. 1982, 86, 5145-5150. [CrossRef]

38. Moschovas, D.; Manesi, G.-M.; Karydis-Messinis, A.; Zapsas, G.; Ntetsikas, K.; Zafeiropoulos, N.E.; Piryazev, A.A.; Thomas, E.L.; Hadjichristidis, N.; Ivanov, D.A.; et al. Alternating Gyroid Network Structure in an ABC Miktoarm Terpolymer Comprised of Polystyrene and Two Polydienes. Nanomaterials 2020, 10, 1497. [CrossRef] [PubMed]

39. Feng, X.; Zhuo, M.; Guo, H.; Thomas, E.L. Visualizing the double-gyroid twin. Proc. Natl. Acad. Sci. USA 2021, 118, e2018977118. [CrossRef] [PubMed] 